Article

\title{
The Appropriate Opportunity for Evaluating Liver Fibrosis by Using the FIB-4 Index in Patients with Nonalcoholic Fatty Liver Disease in Japan
}

\author{
Yuya Seko ${ }^{1, *(1)}$, Kota Yano ${ }^{1}$, Aya Takahashi ${ }^{1}$, Shinya Okishio ${ }^{1}$, Seita Kataoka ${ }^{1}$, \\ Keiichiroh Okuda ${ }^{1}$, Atsushi Umemura ${ }^{1}$, Kanji Yamaguchi ${ }^{1}$, Michihisa Moriguchi ${ }^{1}$, \\ Saiyu Tanaka ${ }^{2}$ and Yoshito Itoh ${ }^{1}$ \\ 1 Department of Molecular Gastroenterology and Hepatology, Kyoto Prefectural University of Medicine, \\ Kyoto 6028566, Japan; yanokota@koto.kpu-m.ac.jp (K.Y.); ayataka@koto.kpu-m.ac.jp (A.T.); \\ okishin@koto.kpu-m.ac.jp (S.O.); s1120@koto.kpu-m.ac.jp (S.K.); k-okuda@koto.kpu-m.ac.jp (K.O.); \\ aumemura@koto.kpu-m.ac.jp (A.U.); ykanji@koto.kpu-m.ac.jp (K.Y.); mmori@koto.kpu-m.ac.jp (M.M.); \\ yitoh@koto.kpu-m.ac.jp (Y.I.) \\ 2 Center for Digestive and Liver Diseases, Nara City Hospital, Nara 6308305, Japan; saiyusun@yahoo.co.jp \\ * Correspondence: yuyaseko@koto.kpu-m.ac.jp; Tel.: +81-75-251-5519
}

Received: 2 September 2020; Accepted: 19 October 2020; Published: 19 October 2020

check for updates

\begin{abstract}
In patients with nonalcoholic fatty liver disease (NAFLD), liver fibrosis is the predictive factor for liver-related events and prognosis. This retrospective study aimed to evaluate longitudinal changes in the FIB-4 index and to determine a strategy for diagnosing and following patients with NAFLD using this index. We analyzed the FIB-4 index at baseline and after 1 and 5 years in 272 consecutive patients with biopsy-proven NAFLD. Of these, 52 patients underwent serial biopsies. The change in the FIB-4 index was correlated with changes in the fibrosis stage among these patients $(p=0.048)$. The median FIB- 4 index was 1.64 at baseline, 1.45 at 1 year, and 1.74 at 5 years. The negative predictive value for advanced fibrosis at a low cutoff point was 90.4/90.1 at baseline/ 1 year. Its specificity at a high cutoff point increased from $65.0 \%$ at baseline to $82.3 \%$ at 1 year. Multivariate analysis identified the FIB- 4 index at 1 year as a predictive factor for a FIB- 4 index $>2.67$ at 5 years. A FIB-4 index $<1.30$ was acceptable for excluding advanced fibrosis at baseline. In contrast, to evaluate and predict advanced liver fibrosis with the FIB-4 index at a high cutoff point, we should use the index at 1 year after appropriate therapy.
\end{abstract}

Keywords: FIB-4 index; nonalcoholic fatty liver disease; liver fibrosis

\section{Introduction}

Nonalcoholic fatty liver disease (NAFLD) is the most common chronic liver disease in Japan, and it affects up to $25-30 \%$ of the general adult population worldwide [1]. NAFLD is one of the phenotypes of metabolic syndrome in the liver, and it encompasses a wide spectrum of liver pathology, ranging from non-alcoholic fatty liver (NAFL), which is usually benign, to non-alcoholic steatohepatitis (NASH), which is characterized by steatosis, lobular inflammation, and hepatocellular injury, and may progress to liver cirrhosis (LC), hepatic failure, and hepatocellular carcinoma (HCC) in the absence of significant alcohol consumption. A recent large cohort study reported that hepatic fibrosis was the only predictive factor of not only liver-related events but also the mortality of patients with NAFLD [2,3]. These results indicate that accurate staging of fibrosis is clinically important for patients with NASH to prevent severe complications. Patients with NAFLD, particularly those with advanced symptoms, are at high risk for HCC and death from liver-related causes. Previous studies reported that 27.0-37.6\% of patients with NAFLD experience disease progression [4-11]. Bodyweight gain, high insulin resistance, type 2 
diabetes mellitus (T2DM), and low initial fibrosis stage are correlated with fibrosis progression among NAFLD patients [5-7]. Among NASH patients, obesity, change in alanine aminotransferase (ALT) levels, and weight gain are associated with progression [8,9]. One previous meta-analysis of serial biopsy studies reported that lobular inflammation at first biopsy predicted progression to advanced fibrosis [10]. However, no reports of noninvasive fibrosis score have clarified the predictive factors associated with histological changes in Japanese patients with biopsy-proven NAFLD.

Liver biopsy remains the gold standard for the diagnosis of NASH. However, liver biopsy is invasive, and has several limitations, such as sampling error and cost [12-14]. Intraobserver and interobserver variability are also potential concerns. In the previous study by Kleiner et al., eight pathologists reviewed the biopsy specimens twice. The intraobserver agreement rate for fibrosis was 0.85 , and the interobserver agreement rate was 0.84 [15]. Ratziu et al. estimated the sampling error and intraobserver difference by collecting two specimens from each patient and having one pathologist review the specimens on two separate occasions, 3 months apart. There was at least a one-stage difference between the two specimens in $41 \%$ of patients. The reliability rate was only 0.47 [16]. Fibrosis variability was present not only in the early stage but also in the advanced stage. Non-invasive approaches to identify the stage of liver fibrosis have been devised with a combination of clinical parameters and imaging methods, including transient elastography (TE) or magnetic resonance elastography (MRE). However, obesity can be a reason for failed TE, and MRE is still expensive and not widely available. The diagnostic accuracy of early stage NASH (stage 1) from stages 2-4 is still unclear for both TE and MRE methods. The Fibrosis-4 (FIB-4) index is a noninvasive fibrosis score based on blood parameters measured routinely, such as aspartate aminotransferase (AST), ALT, and platelet count, as well as age. According to some studies, the performance of the FIB- 4 index for the diagnosis of advanced fibrosis in NAFLD is better than that of other non-invasive parameters in both Caucasian and Japanese subjects [17,18]. However, the FIB-4 index often changes in the follow-up period, and its accuracy for detecting advanced fibrosis is not high enough. Furthermore, recent large cohort study in the general population reported that biomarkers including the FIB-4 index have limitations on their ability to evaluate liver fibrosis because of underlying disease. They found only modest associations between liver stiffness measured by TE and biomarkers of liver fibrosis [19]. There is a paucity of longitudinal follow-up data regarding changes in the FIB-4 index in patients with NAFLD.

In this study, we therefore aimed to clarify the accuracy of the FIB-4 index at baseline and after appropriate therapy in the follow-up period, and to determine a strategy for detecting advanced fibrosis in patients with NAFLD using the FIB-4 index.

\section{Materials and Methods}

\subsection{Patients}

A total of 272 consecutive patients who underwent liver biopsy and were diagnosed with NAFLD at the Department of Gastroenterology and Hepatology, Kyoto Prefectural University of Medicine (Kyoto, Japan), and Center for Digestive and Liver Disease in Nara City Hospital (Nara, Japan) from January 2005 to March 2015 were enrolled in this retrospective study. All patients were followed up with for more than 5 years. We diagnosed NAFLD on the basis of liver biopsy findings of steatosis in at least $5 \%$ of hepatocytes and the exclusion of other liver diseases, including viral hepatitis, autoimmune hepatitis, and drug-induced liver disease. Patients with a daily alcohol consumption greater than $30 \mathrm{~g}$ for men and greater than $20 \mathrm{~g}$ for women were excluded. Of these, 52 patients underwent serial biopsies. The median interval between basal and second liver biopsies was 968 days (210-2778 days). Patients were given medications as necessary during the follow-up period. This study was approved by the Ethical Review Board of Kyoto Prefectural University of Medicine (ERB-C-544-2), approved on 10, July, 2012. Review Board of Nara City Hospital (No. 35), approved on 10 January 2016. All patients provided written informed consent at the time of liver biopsy, and the study was conducted in accordance with the 2013 Declaration of Helsinki. 


\subsection{Laboratory and Clinical Parameters}

Venous blood samples were collected the morning after a 12-h overnight fast. Laboratory assays included blood cell counts and measurements of serum concentrations of AST, ALT, gamma glutamyl transpeptidase (GGT), total cholesterol, high-density lipoprotein (HDL) cholesterol, low-density lipoprotein (LDL) cholesterol, triglycerides, fasting plasma glucose (FPG), and type IV collagen 7s. These parameters were measured with standard clinical chemistry laboratory techniques. Body mass index (BMI) was calculated as weight in kilograms/(height in meters) $)^{2}$. T2DM was diagnosed according to the Report of the Expert Committee on the Diagnosis and Classification of Diabetes Mellitus or based on the administration of anti-T2DM agents. Patients with serum cholesterol concentrations $>220 \mathrm{mg} / \mathrm{dl}$ or triglyceride concentrations $>160 \mathrm{mg} / \mathrm{dl}$ or who were receiving treatment with anti-dyslipidemia agents were defined as having dyslipidemia. The Fibrosis-4 (FIB-4) index was calculated as follows: $\left([\right.$ age $($ years $) \times$ AST $(\mathrm{IU} / \mathrm{L})] /$ platelet count $\left.\left[10^{9} / \mathrm{L}\right]\right) \times(\text { ALT }[\mathrm{IU} / \mathrm{L}])^{1 / 2}$.

\subsection{Liver Histology}

All enrolled patients underwent a percutaneous liver biopsy under ultrasonic guidance. The liver specimens were embedded in paraffin and stained with hematoxylin and eosin and Masson-trichrome. The specimens were evaluated by two hepatic pathologists who were blinded to the clinical findings. An adequate liver biopsy sample was defined as a specimen $>1.5 \mathrm{~cm}$ long and/or having more than 11 portal tracts. NASH was defined as steatosis with lobular inflammation and ballooning degeneration, with or without Mallory-Denk bodies or fibrosis. Patients with liver biopsy specimens that showed simple steatosis or steatosis with non-specific inflammation were diagnosed with NAFL. Specimens with steatosis of $<5,5-33,>33-66$, or $>66 \%$ were scored as steatosis grades $0,1,2$, and 3 , respectively. For mild, moderate, and severe ballooning and inflammation (acinar and portal) the necroinflammatory grades were 1,2, and 3, respectively. The severity of hepatic fibrosis (stage) was scored as: stage 1 , zone 3 perisinusoidal fibrosis; stage 2, zone 3 perisinusoidal fibrosis with portal fibrosis; stage 3 , zone 3 perisinusoidal fibrosis and portal fibrosis with bridging fibrosis; and stage 4 , cirrhosis.

\subsection{DNA Preparation and SNP Genotyping}

Genomic DNA was extracted from blood samples via the DNeasy Blood \& Tissue kit (Qiagen, Tokyo, Japan). The SNP rs738409 was genotyped in each sample using TaqMan SNP genotyping assays (Applied Biosystems, Foster City, CA, USA) with commercially available predesigned SNP-specific primers for PCR amplification and extension reactions according to the manufacturer's protocol. The precise protocol was performed in a manner similar to that in our previous study $[20,21]$.

\subsection{Statistical Analysis}

The distribution of subject characteristics was assessed by the chi-square test or Mann-Whitney's $\mathrm{U}$ test, as appropriate. To assess the accuracy of the FIB-4 index in differentiating advanced fibrosis, we calculated the sensitivity (Se), specificity (Sp), positive predictive value (PPV), and negative predictive value (NPV) for the cut-off values $(<1.30$ and $>2.67)$ proposed by Shah [18]. The dependent data was assessed by using the Wilcoxon signed-rank test. We compared fibrosis stage between first and second biopsy, and the change in fibrosis stage was divided by the interval period between basal and second biopsy in 52 patients. We performed logistic regression analysis to evaluate predictive factors of the FIB-4 index at 5 years adjusted for sex (male, female), age, BMI, presence of T2DM, hypertension, hyperlipidemia (yes, no), PNPLA3 (CC, CG, GG), and FIB-4 index at baseline and 1 year. All reported $P$ values were two-sided, and the significance level was set at 0.05 . Statistical comparisons were performed with SPSS software (version 25, SPSS Inc., Chicago, IL, USA). 


\section{Results}

\subsection{Patient Characteristics}

A total of 272 patients with NAFLD were analyzed. Table 1 summarizes the demographic profiles and laboratory and histologic data of the study patients at baseline and 5 years later. The median age was 60 years, 122 patients (44.9\%) were men, 137 patients (50.4\%) were diagnosed with T2DM, 184 (67.6\%) had hypertension, and 184 (67.6\%) had hyperlipidemia. Forty-six patients had advanced fibrosis (stage 3 or 4) including 16 patients with cirrhosis. There was a significant decrease in BMI and albumin, AST, ALT, GGT, and LDL-cholesterol levels from baseline to 5 years after treatment in the overall population. The mean FIB-4 indices for stages $0,1,2,3$, and 4 were 1.20, 1.59, 2.08, 2.44, and 3.31, respectively ( $p<0.0001$ by analysis of variance). The mean FIB- 4 index was 1.55 in patients with stage $0-2$ fibrosis and 2.72 in patients with stage $3-4$ fibrosis $(p<0.001)$. Table 2 summarizes the demographic profiles and laboratory and histological data of study patients at initial and second biopsy. During the follow-up period, significant reductions in albumin, AST, ALT, $\gamma$-GT, platelet count, triglyceride, and type IV collagen 7s were observed. At initial biopsy, 14 patients had advanced fibrosis. At the second biopsy, 11 patients had advanced fibrosis, including 3 patients which had progressed to stage 4 .

Table 1. Characteristics of 272 patients with nonalcoholic fatty liver disease at baseline and 5 years later.

\begin{tabular}{ccccc}
\hline Variable $\boldsymbol{n}=\mathbf{2 7 2}$ & Baseline & 1 Year & 5 Years & $\begin{array}{c}p \\
\text { (Baseline and 5 Years) }\end{array}$ \\
\hline Sex, male & $122(44.9 \%)$ & & & \\
Age, years & $60(21-83)$ & & & \\
PNPLA3, CC/CG/GG & $35 / 93 / 87$ & & & \\
Hyperlipidemia & $184(67.6 \%)$ & & & \\
T2DM & $137(50.4 \%)$ & & & \\
Hypertension & $112(41.2 \%)$ & & & \\
BMI, kg/m 2 & $26.9(18.9-43.0)$ & $26.5(17.2-43.0)$ & $26.4(17.4-46.2)$ & $<013$ \\
Albumin, g/dL & $4.4(3.1-5.3)$ & $4.4(3.0-5.6)$ & $4.3(2.5-5.6)$ & $<0.001$ \\
AST, IU/L & $44(12-217)$ & $32(14-166)$ & $31(11-158)$ & 0.001 \\
ALT, IU/L & $62(7-266)$ & $38(4-278)$ & $34(3-180)$ & 0.089 \\
GGT, IU/L & $61(14-533)$ & $47(15-415)$ & $39(8-405)$ & 0.061 \\
Platelet count, $\times 10^{3} / \mu L$ & $213(46-454)$ & $217(46-393)$ & $207(25-433)$ & 0.179 \\
Total cholesterol, mg/dL & $202(66-350)$ & $202(74-334)$ & $198(87-301)$ & 0.490 \\
Triglycerides, mg/dL & $146(18-739)$ & $140(32-692)$ & $132(37-593)$ & $<0.001$ \\
LDL-C, mg/dL & $124(44-301)$ & $120(48-286)$ & $118(38-232)$ & 0.099 \\
HDL-C, mg/dL & $54(21-151)$ & $54(23-138)$ & $54(23-134)$ & 0.557 \\
FPG, mg/dL & $102(60-420)$ & $106(68-258)$ & $111(62-279)$ & \\
FIB-4 index & $1.64(0.31-9.84)$ & $1.45(0.32-11.73)$ & $1.74(0.31-15.86)$ & \\
Type IV collagen 7s, ng/mL & $4.6(2.6-11.0)$ & $4.8(2.8-17.0)$ & $4.8(1.6-17.0)$ & \\
Fibrosis stage $(0 / 1 / 2 / 3 / 4)$ & $83 / 99 / 44 / 30 / 16$ & & & \\
\hline
\end{tabular}

Results are presented as $\mathrm{n}(\%)$ for qualitative data or as median (range) for quantitative data. Abbreviations: T2DM, type 2 diabetes mellitus; BMI, body mass index; AST, aspartate aminotransferase; ALT, alanine aminotransferase; GGT, gamma-glutamyl transferase; LDL-C, low-density lipoprotein cholesterol; HDL-C, high-density lipoprotein cholesterol; FIB-4, Fibrosis-4; FPG; fasting plasma glucose.

Table 2. Characteristics of 52 patients with nonalcoholic fatty liver disease at the initial and second biopsies.

\begin{tabular}{cccc}
\hline Variable $\boldsymbol{n}=\mathbf{5 2}$ & At Initial Biopsy & At Second Biopsy & $p$ \\
\hline Sex, male & $22(42.3 \%)$ & & \\
Age, years & $68(21-83)$ & & \\
Hyperlipidemia & $33(63.5 \%)$ & & \\
T2DM & $26(50.0 \%)$ & & \\
Hypertension & $24(46.2 \%)$ & $26.6(19.0-49.5)$ & 0.486 \\
BMI, kg/m ${ }^{2}$ & $26.4(18.9-43.0)$ & $4.1(3.4-5.1)$ & 0.001 \\
Albumin, g/dL & $4.3(3.2-4.9)$ & $38(15-211)$ & $<0.001$ \\
AST, IU/L & $57(22-186)$ & & \\
\hline
\end{tabular}


Table 2. Cont.

\begin{tabular}{cccc}
\hline Variable $\boldsymbol{n}=\mathbf{5 2}$ & At Initial Biopsy & At Second Biopsy & $p$ \\
\hline Platelet count, $\times 10^{3} / \mathrm{\mu L}$ & $207(105-417)$ & $195(93-403)$ & 0.040 \\
Total cholesterol, $\mathrm{mg} / \mathrm{dL}$ & $201(117-292)$ & $191(93-436)$ & 0.203 \\
Triglycerides, $\mathrm{mg} / \mathrm{dL}$ & $153(71-559)$ & $130(60-351)$ & 0.022 \\
LDL-C, $\mathrm{mg} / \mathrm{dL}$ & $104(43-225)$ & $124(43-209)$ & 0.035 \\
HDL-C, $\mathrm{mg} / \mathrm{dL}$ & $50(22-85)$ & $51(28-95)$ & 0.748 \\
FPG, mg/dL & $94(60-260)$ & $96(65-182)$ & 0.664 \\
FIB-4 index & $2.12(0.31-9.04)$ & $2.20(0.26-3.14)$ & 0.973 \\
Type IV collagen $7 \mathrm{~s}, \mathrm{ng} / \mathrm{mL}$ & $5.3(2.7-13.0)$ & $4.4(2.6-9.5)$ & 0.002 \\
Fibrosis stage $(0 / 1 / 2 / 3 / 4)$ & $0 / 24 / 14 / 14 / 0$ & $2 / 18 / 21 / 8 / 3$ & \\
ALT, IU/L & $83(20-358)$ & $46(7-251)$ & $<0.001$ \\
GGT, IU/L & $73(22-306)$ & $45(8-316)$ & 0.009 \\
\hline
\end{tabular}

Abbreviations are defined in Table 1.

\subsection{Correlation between Histological Change and Change in FIB-4 Index}

Among 52 patients, 13 patients ( $25 \%$ ) had fibrosis progression of one stage or more, the stage remained unchanged in 26 patients $(50 \%)$, and 13 patients (25\%) showed fibrosis regression (Table 3 ). The change amounts of each fibrosis stage were ranged from -2 to 2 stages, and from -1.38 stage/year to 1.62 stage/year. The overall annual rate of fibrosis change was 0.002/year. Figure 1 shows the correlation between change in liver fibrosis stage per year and the FIB-4 index. There was significantly weak positive correlation between change in fibrosis stage and the FIB-4 index $(p=0.048, r=0.265)$. Among 23 patients with a worse FIB-4 index result from baseline to the second biopsy, the fibrosis stage progression rate was 0.10 stage/year, while that of 29 patients with a better FIB-4 index result was -0.08 stage/year. Other fibrosis markers, such as platelet count and Type IV collagen $7 \mathrm{~s}$ did not show a significant correlation.

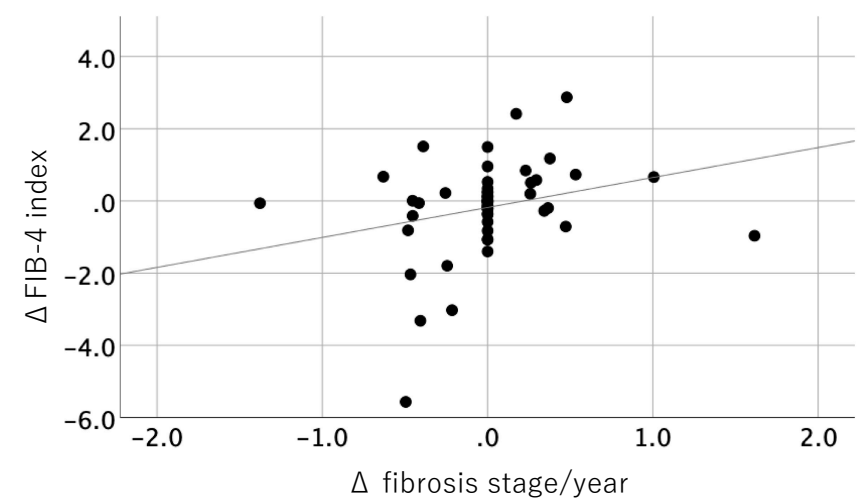

Figure 1. Correlation between changes in the FIB-4 index and change in fibrosis stage per year.

Table 3. Distribution of fibrosis stage at initial and second biopsies.

\begin{tabular}{ccccccc}
\hline & \multicolumn{7}{c}{ Stage at Second Biopsy } \\
\hline & 0 & 1 & 2 & 3 & 4 & Total \\
\hline Stage at initial biopsy & & & & & & \\
\hline 1 & 2 & 13 & 7 & 2 & 0 & 24 \\
2 & 0 & 4 & 8 & 1 & 1 & 14 \\
3 & 0 & 1 & 6 & 5 & 2 & 14 \\
\hline Total & 2 & 18 & 21 & 8 & 3 & 52 \\
\hline
\end{tabular}




\subsection{Changes in the FIB-4 Index During the Follow-Up Period and Accuracy for Advanced Fibrosis}

We divided subjects into three groups by cut-off values $(<1.30,1.30-2.67$, and $>2.67)$. The proportion of patients within each group (high/intermediate/low) was $39.7 \% / 29.8 \% / 30.5 \%$ at baseline and $23.5 \% / 44.1 \% / 32.4 \%$ at 5 years. The number of patients with a high FIB-4 index decreased from 108 at baseline to 64 at 5 years. The median FIB- 4 index decreased significantly from 1.64 at baseline to 1.45 at 1 year $(p<0.001)$ and increased to 1.74 at 5 years (Figure 2a). Regarding the components of the FIB-4 index, the serum levels of AST and ALT decreased significantly from baseline to 1 year $(p<0.001)$. The level of ALT also decreased significantly from 1 year to 5 years $(p=0.017)$, while AST did not change significantly $(p=0.189)$. Platelet counts increased from baseline to 1 year, then decreased significantly from 1 year to 5 years $(p=0.007)$ (Figure $2 b)$. Table 4 shows the number of patients according to the fibrosis stage at baseline and 1 year. Among the 46 patients with advanced fibrosis, 8 and 11 patients had a FIB- 4 index result of $<1.30$ at baseline and at 1 year, respectively. Among 29 patients at stage 3,4 who had FIB- $4>2.67$ at baseline, 20 patients $(69.0 \%)$ showed a FIB- $4>2.67$ at 1 year too. On the other hand, among 79 patients at stages 1,2 and with FIB- $4>2.67$ at baseline, only 40 patients $(50.6 \%)$ showed FIB-4 $>2.67$ at 1 year (Table 4 ). We calculated the sensitivity and specificity of the FIB- 4 index at baseline and after 1 year for differentiating baseline advanced fibrosis from non-advanced fibrosis. At a low cut-off value, the sensitivity, specificity, positive predictive value (PPV), and negative predictive value (NPV) were $82.6 \% / 33.2 \% / 20.1 \% / 90.4 \%$, respectively, at baseline, and 76.9\%/44.2\%/21.7\%/90.1\%, respectively, at 1 year. At a high cut-off value, those values were $63.0 \% / 65.0 \% / 26.9 \% / 89.6 \%$, respectively, at baseline and $43.4 \% / 82.3 \% / 33.3 \% / 87.7 \%$, respectively, at 1 year (Table 4 ).

(a)

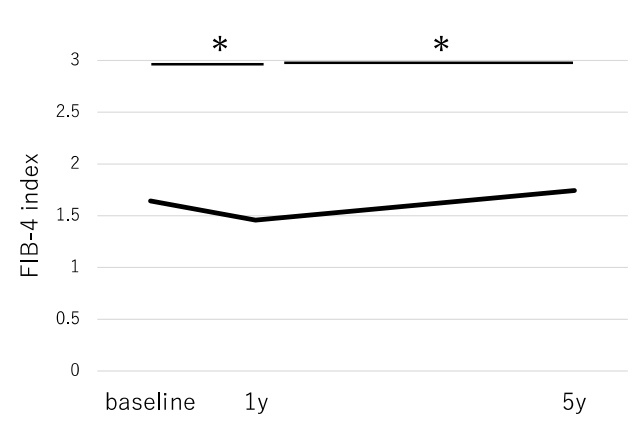

(b)

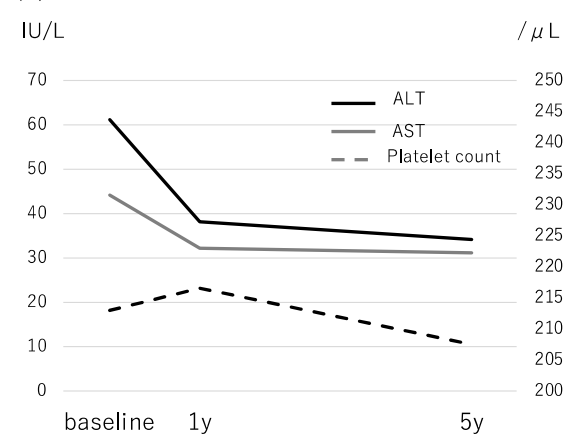

Figure 2. Changes in (a) the FIB-4 index and (b) AST, ALT, and platelet count in the follow-up period. $* p<0.05$.

Table 4. (a) Number of patients according to baseline fibrosis stage and (b) accuracy of the FIB-4 index at baseline and after 1 year.

\begin{tabular}{|c|c|c|c|c|c|}
\hline \multicolumn{6}{|l|}{ (a) } \\
\hline & \multicolumn{2}{|c|}{ Baseline } & \multicolumn{3}{|c|}{1 Year } \\
\hline & Stage 3,4 & Stage 0-2 & Stage 3,4 & \multicolumn{2}{|c|}{ Stage 0-2 } \\
\hline$<1.30$ & 8 & 75 & 11 & \multicolumn{2}{|c|}{100} \\
\hline$\geqq 1.30$ & 38 & 151 & 35 & \multicolumn{2}{|c|}{126} \\
\hline$>2.67$ & 17 & 147 & 26 & \multicolumn{2}{|c|}{186} \\
\hline$\leqq 2.67$ & 29 & 79 & 20 & \multicolumn{2}{|c|}{40} \\
\hline \multicolumn{6}{|l|}{ (b) } \\
\hline & & Sensitivity & Specificity & PPV & NPV \\
\hline \multirow{2}{*}{ Baseline } & Low cut-off & 82.6 & 33.2 & 20.1 & 90.4 \\
\hline & High cut-off & 63.0 & 65.0 & 26.9 & 89.6 \\
\hline \multirow{2}{*}{1 year } & Low cut-off & 76.9 & 44.2 & 21.7 & 90.1 \\
\hline & High cut-off & 43.4 & 82.3 & 33.3 & 87.7 \\
\hline
\end{tabular}

Abbreviations: PPV, positive predictive value; NPV, negative predictive value. 


\subsection{Factors Associated with a FIB-4 Index $>2.67$ at 5 Years}

Among 272 patients with NAFLD, the number of patients with a high FIB-4 index decreased from $108(39.7 \%)$ at baseline to $64(23.5 \%)$ at 5 years. We performed multivariate analysis using sex, age, hypertension, T2DM, hyperlipidemia, PNPLA3 genotype, the FIB-4 index at baseline, and FIB-4 index at 1 year as factors (Table 5). The analysis identified FIB-4 index at 1 year $(>2.67$, odds ratio [OR] 41.65, $p<0.01$ ) as a predictive factor for a FIB- 4 index $>2.67$ at 5 years. On the other hand, the FIB- 4 index at baseline did not predict a high FIB-4 index at 5 years. Among the 108 patients with a high FIB-4 index at baseline, $51(47.2 \%)$ had a FIB-4 index $>2.67$ at 5 years. Among 60 patients with a high FIB-4 index at 1 year, $49(81.7 \%)$ had a FIB- 4 index $>2.67$ at 5 years.

Table 5. Factors associated with FIB- $4>2.67$ at 5 year in patients with non-alcoholic fatty liver disease based on univariate and multivariate analysis.

\begin{tabular}{|c|c|c|c|c|}
\hline Variable & Category & $p$ Value & OR $(95 \% \mathrm{CI})^{\mathrm{a}}$ & $p$ Value \\
\hline Age, year & Per 1 year & & $1.05(0.99-1.11)$ & 0.086 \\
\hline BMI, $\mathrm{kg} / \mathrm{m}^{2}$ & Per $1 \mathrm{~kg} / \mathrm{m}^{2}$ & & $0.97(0.86-1.11)$ & 0.679 \\
\hline Sex & $\begin{array}{c}\text { 1: male } \\
\text { 2: female }\end{array}$ & 0.002 & $0.34(0.11-1.05)$ & 0.060 \\
\hline $\mathrm{T} 2 \mathrm{DM}$ & $\begin{array}{l}\text { 1: no } \\
2: \text { yes }\end{array}$ & 0.089 & $0.86(0.31-2.36)$ & 0.765 \\
\hline PNPLA3 & $\begin{array}{l}\text { 1: } \mathrm{CC} \\
\text { 2: } \mathrm{CG} \\
\text { 3: GG }\end{array}$ & $\begin{array}{l}0.088 \\
0.040\end{array}$ & $\begin{array}{c}1 \\
1.89(0.31-11.41) \\
4.50(0.74-27.32)\end{array}$ & $\begin{array}{l}0.490 \\
0.102\end{array}$ \\
\hline FIB-4 index at baseline & $\begin{array}{l}1:-2.67 \\
2:>2.67\end{array}$ & $<0.01$ & $2.61(0.88-7.69)$ & 0.082 \\
\hline FIB- 4 index at 1 year & $\begin{array}{l}1:-2.67 \\
2:>2.67\end{array}$ & $<0.01$ & $41.65(13.11-132.33)$ & $<0.01$ \\
\hline
\end{tabular}

Abbreviations are defined in Table 1. OR: odds ratio, CI: confidence interval. a Estimated using logistic regression analysis.

\section{Discussion}

Staging of liver fibrosis in patients with NAFLD is essential for stratifying patients according to prognosis, treatment strategy, and guiding surveillance for the development of HCC. Because of the large number of patients with NAFLD, it is impossible to perform liver biopsy in all patients. Non-invasive fibrosis assessments are widely developed for the selection of patients considered to require further inspection. It is still controversial whether a non-invasive fibrosis score is preferred to elastography or not. Elastography has high diagnostic performance in noninvasive detection of liver fibrosis and steatosis in patients with NAFLD, especially with measuring spleen stiffness [22,23]. However, Liver stiffness measurement by TE is reported to be overestimated by transaminases flare [24]. Several studies suggested that FIB-4 index is considered to be one of the most useful scoring systems, especially combined with ultrasound and MRI-based elastography $[25,26]$. Sumida et al. reported that the FIB-4 index had a high NPV for excluding advanced fibrosis and was superior to other scoring systems [17]. The FIB-4 index also has several concerns. Whether the changes in the FIB-4 index during the follow-up period truly reflect a change in fibrosis is unclear. EASL-ALEH clinical practice guidelines reported that a FIB-4 index assessment may be less sensitive in detecting changes in intermediate stages of fibrosis [27]. One of the reasons for that concern is the components of the FIB-4 index. The serum levels of AST and ALT change drastically with diet and exercise therapy. Several treatments for complications of NAFLD, such as T2DM and hyperlipidemia, also decrease transaminase levels. Sodium glucose cotransporter 2 inhibitors and glucagon-like peptidase- 1 receptor agonist use for T2DM can decrease transaminase activity in patients with NAFLD [27-30]. Statin use 
for hyperlipidemia was reported to have a protective effect against liver damage in patients with NAFLD [31]. It is also unknown whether the FIB-4 index can become a surrogate marker for evaluating histological changes, because longitudinal studies about the correlation between change in FIB-4 index and fibrosis stage are rarely performed.

In the present study, we first clarified the significance of determining the FIB-4 index to estimate fibrosis progression by serial biopsies study. The change in FIB-4 index was significantly correlated with change in fibrosis stage in Japanese patients with NAFLD. Our result indicate that serial FIB-4 index measurements are relevant for monitoring histological changes and have potential for good index in the treatment of NAFLD. The post hoc analysis of FLINT study revealed that a reduction in the FIB-4 index at 24 weeks significantly correlated with improvement of fibrosis stage at 72 weeks [32]. This report supported our results.

The median level of AST/ALT decreased from 44/62 at baseline to 32/38 at 1 year and 31/34 at 5 years. It is hard to believe that fibrosis was ameliorated after 1 year of treatment. We previously reported that the average annual rate of progression of liver fibrosis was 0.002 stages per year in treated patients with NASH [33]. A meta-analysis of serial biopsy studies in mostly untreated patients with NAFLD identified the rate of fibrosis change to be 0.07 stages/year in NAFL and 0.14 stages/year in NASH [34]. Based on these studies, the fibrosis stage at 1 year after baseline may not change from that of baseline. The accuracy of the FIB-4 index in this study was worse than that in a previous study in Japanese patients [17]. A plausible reason for this difference may be the difference in cut-off value and the proportion of patients with advanced-stage disease. In this study, the accuracy of the FIB-4 index differed between baseline and 1 year. The NPV for a low cut-off value $(<1.30)$ was about $90 \%$ and did not change between the two points. Regarding the high cut-off value $(>2.67)$, among 108 patients who had a FIB-4 index $>2.67$ at baseline, 79 (73.1\%) had non-advanced fibrosis NAFLD. At 1 year, 40 of 60 patients $(66.7 \%)$ who had a FIB- 4 index $>2.67$ were without advanced fibrosis. The specificity for diagnosing advanced fibrosis increased from $65 \%$ at baseline to $82.3 \%$ at 1 year. This result suggests that when we try to find patients with advanced fibrosis by using a high cut-off of the FIB- 4 index, we should diagnose them after adequate therapy for NAFLD. We also could avoid unnecessary liver biopsy by using high cut-off of FIB- 4 index at 1 year. The FIB- 4 index at 1 year after baseline was also identified as a predictive factor for a FIB- 4 index $>2.67$ at 5 years after baseline. This finding suggests that early changes in the FIB-4 index may help us in predicting patients with therapy resistance and progression of fibrosis. These patients may need serial liver biopsy to confirm the change in histological findings and to determine changes in treatment or surveillance plan.

This study has several limitations. It was a retrospective study. The number of subjects, especially with advanced fibrosis, was smaller than in previous Asian studies [17,35]. Further large-scale studies using serial liver biopsy will be required to draw firm conclusions about the relationship between the FIB-4 index and liver fibrosis. Referral bias and patient selection bias could have existed because liver biopsy might have been considered for patients with NAFLD who were likely to have liver fibrosis.

In conclusion, the NPV of a FIB-4 index $<1.30$ was not different between baseline and 1 year; thus, it was acceptable to exclude advanced fibrosis at baseline. On the other hand, to evaluate and predict advanced liver fibrosis using a FIB-4 index $>2.67$, we should use the index at 1 year after appropriate therapy. Taking the right opportunity for evaluating liver fibrosis by using the FIB-4 index seems to be important for excluding or predicting advanced fibrosis and avoiding unnecessary liver biopsies.

Author Contributions: Conceptualization, Y.S. and Y.I.; methodology, Y.S.; formal analysis, Y.S.; investigation, Y.S.: data curation, Y.S., K.Y. (Kota Yano), A.T., S.O., S.K., K.O., A.U., K.Y. (Kanji Yamaguchi), M.M., S.T. and Y.I.; writing—original draft preparation, Y.S.; writing—review and editing, Y.S., K.Y. (Kota Yano), A.T., S.O., S.K., K.O., A.U., K.Y. (Kanji Yamaguchi), M.M., S.T. and Y.I.; supervision, Y.I.; project administration, Y.S. and Y.I.; funding acquisition, Y.I. All authors have read and agreed to the published version of the manuscript.

Funding: This research received no external funding.

Conflicts of Interest: The authors declare no conflict of interest. 


\section{References}

1. Younossi, Z.M.; Koenig, A.B.; Abdelatif, D.; Fazel, Y.; Henry, L.; Wymer, M. Global epidemiology of nonalcoholic fatty liver disease-Meta-analytic assessment of prevalence, incidence, and outcomes. Hepatology 2016, 64, 73-84. [CrossRef] [PubMed]

2. Chalasani, N.; Younossi, Z.; Lavine, J.E.; Diehl, A.M.; Brunt, E.M.; Cusi, K.; Charlton, M.; Sanyal, A.J. The diagnosis and management of non-alcoholic fatty liver disease: Practice Guideline by the American Association for the Study of Liver Diseases, American College of Gastroenterology, and the American Gastroenterological Association. Hepatology 2012, 55, 2005-2023. [CrossRef] [PubMed]

3. Angulo, P.; Kleiner, D.E.; Dam-Larsen, S.; Adams, L.A.; Bjornsson, E.S.; Charatcharoenwitthaya, P.; Mills, P.R.; Keach, J.C.; Lafferty, H.D.; Stahler, A.; et al. Liver fibrosis, but no other histologic features, is associated with long-term outcomes of patients with nonalcoholic fatty liver disease. Gastroenterology 2015, 149, 389-397. [CrossRef]

4. Pais, R.; Charlotte, F.; Fedchuk, L.; Bedossa, P.; Lebray, P.; Poynard, T.; Ratziu, V.; LIDO Study Group. A systematic review of follow-up biopsies reveals disease progression in patients with non-alcoholic fatty liver. J. Hepatol. 2013, 59, 550-556. [CrossRef] [PubMed]

5. Wong, V.W.; Wong, G.L.; Choi, P.C.; Chan, A.W.; Li, M.K.; Chan, H.Y.; Chim, A.M.; Yu, J.; Sung, J.J.; Chan, H.L. Disease progression of non-alcoholic fatty liver disease: A prospective study with paired liver biopsies at 3 years. Gut 2010, 59, 969-974. [CrossRef]

6. $\quad$ Ekstedt, M.; Franzén, L.E.; Mathiesen, U.L.; Thorelius, L.; Holmqvist, M.; Bodemar, G.; Kechagias, S. Long-term follow-up of patients with NAFLD and elevated liver enzymes. Hepatology 2006, 44, 865-873. [CrossRef] [PubMed]

7. Argo, C.K.; Northup, P.G.; Al-Osaimi, A.M.; Caldwell, S.H. Systematic review of risk factors for fibrosis progression in non-alcoholic steatohepatitis. J. Hepatol. 2009, 51, 371-379. [CrossRef]

8. Fassio, E.; Alvarez, E.; Domínguez, N.; Landeira, G.; Longo, C. Natural history of nonalcoholic steatohepatitis: A longitudinal study of repeat liver biopsies. Hepatology 2004, 40, 820-826. [CrossRef]

9. Hoofnagle, J.H.; Van Natta, M.L.; Kleiner, D.E.; Clark, J.M.; Kowdley, K.V.; Loomba, R.; Neuschwander-Tetri, B.A.; Sanyal, A.J.; Tonascia, J.; Non-alcoholic Steatohepatitis Clinical Research Network (NASH CRN). Vitamin $\mathrm{E}$ and changes in serum alanine aminotransferase levels in patients with non-alcoholic steatohepatitis. Aliment. Pharmacol. Ther. 2013, 38, 134-143. [CrossRef]

10. Adams, L.A.; Sanderson, S.; Lindor, K.D.; Angulo, P. The histological course of nonalcoholic fatty liver disease: A longitudinal study of 103 patients with sequential liver biopsies. J. Hepatol. 2005, 42, 132-138. [CrossRef]

11. Hamaguchi, E.; Takamura, T.; Sakurai, M.; Mizukoshi, E.; Zen, Y.; Takeshita, Y.; Kurita, S.; Arai, K.; Yamashita, T.; Sasaki, M.; et al. Histological course of nonalcoholic fatty liver disease in Japanese patients: Tight glycemic control, rather than weight reduction, ameliorates liver fibrosis. Diabetes Care 2010, 33, $284-286$. [CrossRef] [PubMed]

12. Rockey, D.C.; Caldwell, S.H.; Goodman, Z.D.; Nelson, R.C.; Smith, A.D.; American Association for the Study of Liver Diseases. Liver biopsy. Hepatology 2009, 49, 1017-1044. [CrossRef]

13. Bravo, A.A.; Sheth, S.G.; Chopra, S. Liver biopsy. N. Engl. J. Med. 2001, 344, 495-500. [CrossRef]

14. Vuppalanchi, R.; Ünalp, A.; Van Natta, M.; Cummings, O.W.; Sandrasegaran, K.E.; Hameed, T.; Tonascia, J.; Chalasani, N. Effects of liver biopsy sample length and number of readings on sampling variability in nonalcoholic Fatty liver disease. Clin. Gastroenterol. Hepatol. 2009, 7, 481-486. [CrossRef] [PubMed]

15. Kleiner, D.E.; Brunt, E.M.; Van Natta, M.; Behling, C.; Contos, M.J.; Cummings, O.W.; Ferrell, L.D.; Liu, Y.C.; Torbenson, M.S.; Unalp-Arida, A.; et al. Design and validation of a histological scoring system for nonalcoholic fatty liver disease. Hepatology 2005, 41, 1313-1321. [CrossRef]

16. Ratziu, V.; Charlotte, F.; Heurtier, A.; Gombert, S.; Giral, P.; Bruckert, E.; Grimaldi, A.; Capron, F.; Poynard, T.; LIDO Study Group. Sampling variability of liver biopsy in nonalcoholic fatty liver disease. Gastroenterology 2005, 128, 1898-1906. [CrossRef]

17. Sumida, Y.; Yoneda, M.; Hyogo, H.; Itoh, Y.; Ono, M.; Fujii, H.; Eguchi, Y.; Suzuki, Y.; Aoki, N.; Kanemasa, K.; et al. Validation of the FIB4 index in a Japanese nonalcoholic fatty liver disease population. BMC Gastroenterol. 2012, 5, 12. [CrossRef] [PubMed] 
18. Shah, A.; Lydecker, A.; Murray, K.; Tetri, B.N.; Contos, M.J.; Sanyal, A.J. NASH Clinical Research Network: Comparison of noninvasive markers of fibrosis in patients with nonalcoholic fatty liver disease. Clin. Gastroenterol. Hepatol. 2009, 7, 1104-1112. [CrossRef]

19. Foschi, F.G.; Domenicali, M.; Giacomoni, P.; Dall'Aglio, A.C.; Conti, F.; Borghi, A.; Bevilacqua, V.; Napoli, L.; Mirici, F.; Cucchetti, A.; et al. Is there an association between commonly employed biomarkers of liver fibrosis and liver stiffness in the general population? Ann. Hepatol. 2020, 19, 380-387. [CrossRef]

20. Seko, Y.; Yamaguchi, K.; Mizuno, N.; Okuda, K.; Takemura, M.; Taketani, H.; Hara, T.; Umemura, A.; Nishikawa, T.; Moriguchi, M.; et al. Combination of PNPLA3 and TLL1 polymorphism can predict advanced fibrosis in Japanese patients with nonalcoholic fatty liver disease. J. Gastroenterol. 2018, 53, 438-448. [CrossRef]

21. Nishioji, K.; Mochizuki, N.; Kobayashi, M.; Kamaguchi, M.; Sumida, Y.; Nishimura, T.; Yamaguchi, K.; Kadotani, H.; Itoh, Y. The impact of PNPLA3 rs738409 genetic polymorphism and weight gain $>10 \mathrm{~kg}$ after age 20 on non-alcoholic fatty liver disease in non-obese Japanese individuals. PLoS ONE 2015, 10, e0140427. [CrossRef] [PubMed]

22. Imajo, K.; Kessoku, T.; Honda, Y.; Tomeno, W.; Ogawa, Y.; Mawatari, H.; Fujita, K.; Yoneda, M.; Taguri, M.; Hyogo, H.; et al. Magnetic Resonance Imaging More Accurately Classifies Steatosis and Fibrosis in Patients with Nonalcoholic Fatty Liver Disease Than Transient Elastography. Gastroenterology 2016, 150, 626-637. [CrossRef] [PubMed]

23. Giuffrè, M.; Macor, D.; Masutti, F.; Abazia, C.; Tinè, F.; Patti, R.; Buonocore, M.R.; Colombo, A.; Visintin, A.; Campigotto, M.; et al. Evaluation of spleen stiffness in healthy volunteers using point shear wave elastography. Ann. Hepatol. 2019, 18, 736-741. [CrossRef]

24. Giuffrè, M.; Fouraki, S.; Comar, M.; Masutti, F.; Crocè, L.S. The Importance of Transaminases Flare in Liver Elastography: Characterization of the Probability of Liver Fibrosis Overestimation by Hepatitis C Virus-Induced Cytolysis. Microorganisms 2020, 29, 348. [CrossRef]

25. Yoneda, M.; Imajo, K.; Takahashi, H.; Ogawa, Y.; Eguchi, Y.; Sumida, Y.; Yoneda, M.; Kawanaka, M.; Saito, S.; Tokushige, K.; et al. Clinical strategy of diagnosing and following patients with nonalcoholic fatty liver disease based on invasive and noninvasive methods. J. Gastroenterol. 2018, 53, 181-196. [CrossRef] [PubMed]

26. Davyduke, T.; Tandon, P.; Al-Karaghouli, M.; Abraldes, J.G.; Ma, M.M. Impact of implementing a “FIB-4 First" strategy on a pathway for patients with NAFLD referred from primary care. Hepatol. Commun. 2019, 29, 1322-1333. [CrossRef]

27. European Association for Study of the Liver; Asociación Latinoamericana para el Estudio del Hígado. EASL-ALEH clinical practice guidelines: Non-invasive tests for evaluation of liver disease severity and prognosis. J. Hepatol. 2015, 63, 237-264. [CrossRef] [PubMed]

28. Seko, Y.; Sumida, Y.; Sasaki, K.; Itoh, Y.; Iijima, H.; Hashimoto, T.; Ishii, S.; Inagaki, N. Effects of canagliflozin, an SGLT2 inhibitor, on hepatic function in Japanese patients with type 2 diabetes mellitus: Pooled and subgroup analyses of clinical trials. J. Gastroenterol. 2018, 53, 140-151. [CrossRef] [PubMed]

29. Eguchi, Y.; Kitajima, Y.; Hyogo, H.; Takahashi, H.; Kojima, M.; Ono, M.; Araki, N.; Tanaka, K.; Yamaguchi, M.; Matsuda, Y.; et al. Pilot study of liraglutide effects in non-alcoholic steatohepatitis and non-alcoholic fatty liver disease with glucose intolerance in Japanese patients (LEAN-J). Hepatol. Res. 2015, 45, 269-278. [CrossRef]

30. Seko, Y.; Sumida, Y.; Tanaka, S.; Mori, K.; Taketani, H.; Ishiba, H.; Hara, T.; Okajima, A.; Umemura, A.; Nishikawa, T.; et al. Effect of 12-week dulaglutide therapy in Japanese patients with biopsy-proven non-alcoholic fatty liver disease and type 2 diabetes mellitus. Hepatol. Res. 2017, 47, 1206-1211. [CrossRef] [PubMed]

31. Dongiovanni, P.; Petta, S.; Mannisto, V.; Mancina, R.M.; Pipitone, R.; Karja, V.; Maggioni, M.; Kakela, P.; Wiklund, O.; Mozzi, E.; et al. Statin use and non-alcoholic steatohepatitis in at risk individuals. J. Hepatol. 2015, 63, 705-712. [CrossRef] [PubMed]

32. Chalasani, N.; Abdelmalek, M.F.; Loomba, R.; Kowdley, K.V.; McCullough, A.J.; Dasarathy, S.; Neuschwander-Tetri, B.A.; Terrault, N.; Ferguson, B.; Shringarpure, R.; et al. Relationship between three commonly used non-invasive fibrosis biomarkers and improvement in fibrosis stage in patients with non-alcoholic steatohepatitis. Liver Int. 2019, 39, 924-932. [CrossRef] [PubMed] 
33. Seko, Y.; Sumida, Y.; Tanaka, S.; Mori, K.; Taketani, H.; Ishiba, H.; Hara, T.; Okajima, A.; Yamaguchi, K.; Moriguchi, M.; et al. Serum alanine aminotransferase predicts the histological course of non-alcoholic steatohepatitis in Japanese patients. Hepatol. Res. 2015, 45, E53-E61. [CrossRef] [PubMed]

34. Singh, S.; Allen, A.M.; Wang, Z.; Prokop, L.J.; Murad, M.H.; Loomba, R. Fibrosis progression in nonalcoholic fatty liver vs nonalcoholic steatohepatitis: A systematic review and meta-analysis of paired-biopsy studies. Clin. Gastroenterol. Hepatol. 2015, 13, 643-654. [CrossRef] [PubMed]

35. Wong, V.W.; Wong, G.L.; Chim, A.M.; Tse, A.M.; Tsang, S.W.; Hui, A.Y.; Choi, P.C.; Chan, A.W.; So, W.Y.; Chan, F.K.; et al. Validation of the NAFLD fibrosis score in a Chinese population with low prevalence of advanced fibrosis. Am. J. Gastroenterol. 2008, 103, 1682-1688. [CrossRef] [PubMed]

Publisher's Note: MDPI stays neutral with regard to jurisdictional claims in published maps and institutional affiliations.

(C) 2020 by the authors. Licensee MDPI, Basel, Switzerland. This article is an open access article distributed under the terms and conditions of the Creative Commons Attribution (CC BY) license (http://creativecommons.org/licenses/by/4.0/). 